\title{
Novel TNFAIP3 microdeletion in a girl with infantile-onset inflammatory bowel disease complicated by a severe perianal lesion
}

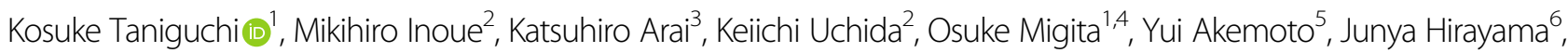 \\ Ichiro Takeuchi ${ }^{3}$, Hirotaka Shimizu ${ }^{3}$ and Kenichiro Hata ${ }^{1}$
}

\begin{abstract}
A20 haploinsufficiency (HA20), a disease caused by loss-of-function TNFAIP3 mutations, manifests various autoinflammatory and/or autoimmune symptoms. Some cases of HA20 were initially diagnosed as very early onset inflammatory bowel disease (VEO-IBD). We performed whole-exome sequencing (WES) for a Japanese girl with infantile-onset IBD and a severe perianal lesion and detected a novel de novo $119 \mathrm{~kb}$ microdeletion containing only TNFAIP3 (arr[GRCh37] 6q23.3(138125829_138244816) × 1).
\end{abstract}

Very early onset inflammatory bowel disease (VEOIBD), defined as IBD with an onset before 6 years of age, is challenging, with difficulties in diagnosis and management. Over 60 genes associated with monogenic IBD have been described worldwide with the widespread use of whole-exome sequencing (WES) ${ }^{1}$. Among them, TNFAIP3 suppresses nuclear factor-kappa $\mathrm{B}$, and its germline mutations lead to $\mathrm{HA} 2 \mathrm{O}^{2}$, presenting with IBD and symptoms similar to those of Behçet disease ${ }^{3}$. However, the diagnostic approach with genetic testing has not been well established, and the genotype-phenotype correlation in HA20 remains unclear. Here, we report a case of infantile-onset HA20 with a severe perianal lesion and a novel de novo microdeletion spanning the TNFAIP3 gene as determined by WES and copy number variation (CNV) analysis.

The patient was an 8-month-old Japanese girl with no consanguinity or other significant family medical history. Her perinatal history was unremarkable. From the age of

\footnotetext{
Correspondence: Kenichiro Hata (hata-k@ncchd.go.jp)

${ }^{1}$ Department of Maternal-Fetal Biology, National Research Institute for Child Health and Development, Tokyo 157-8535, Japan

${ }^{2}$ Department of Gastrointestinal and Pediatric Surgery, Mie University Graduate School of Medicine, Tsu, Mie 514-8507, Japan

Full list of author information is available at the end of the article

These authors contributed equally: Kosuke Taniguchi, Mikihiro Inoue
}

3 weeks, she developed an intermittent high fever every 1-2 months that resolved without intervention. The patient's serum C-reactive protein levels remained elevated, and abdominal computed tomography revealed hepatosplenomegaly. Screening tests previously performed at 1 month of age were not consistent with primary immunodeficiency, autoinflammatory disease, or hemophagocytic syndrome.

The patient started experiencing diarrhea more than ten times a day at 6 months of age, and active perianal fistulae appeared at the age of 7 months. She had impaired growth at 8 months of age, with a height of $63 \mathrm{~cm}(-2.5$ standard deviations (SDs)) and weight of $5.3 \mathrm{~kg}$ ( $-3.0 \mathrm{SDs})$, but was not developmentally delayed. Although she was afebrile, laboratory tests indicated an abnormal condition of the autoimmune system (Supplementary Table S1). The patient had a few aphthous ulcers in the oral cavity, perianal fistulae in the right labia majora and right anterior of the anus, and anal ulcers at the anterior and dorsal sides of the anus (Fig. 1A). Esophagogastroduodenoscopy findings were unremarkable. However, ileocolonoscopy revealed aphthous ulcers throughout the colon with punched-out ulcers over the descending colon to the rectum (Fig. 1B). The terminal ileum was normal. External fistula openings ran directly to the rectal ulcers just 
A

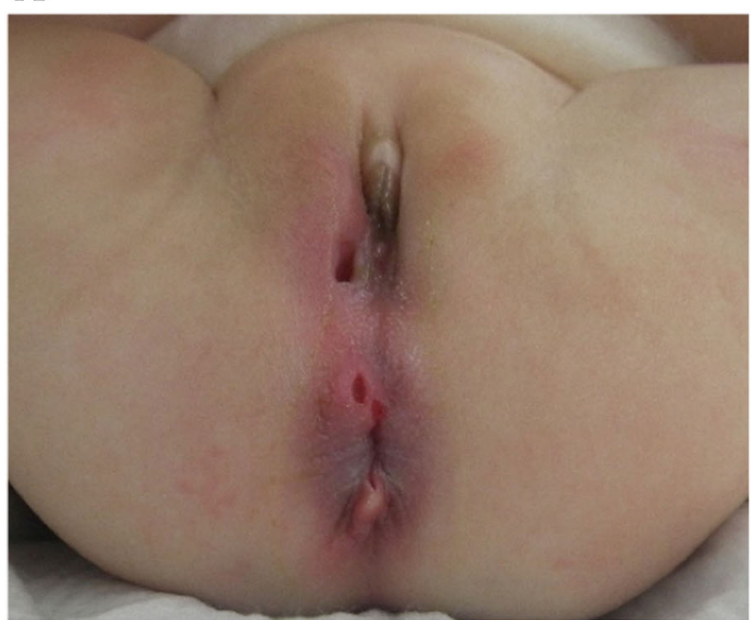

C

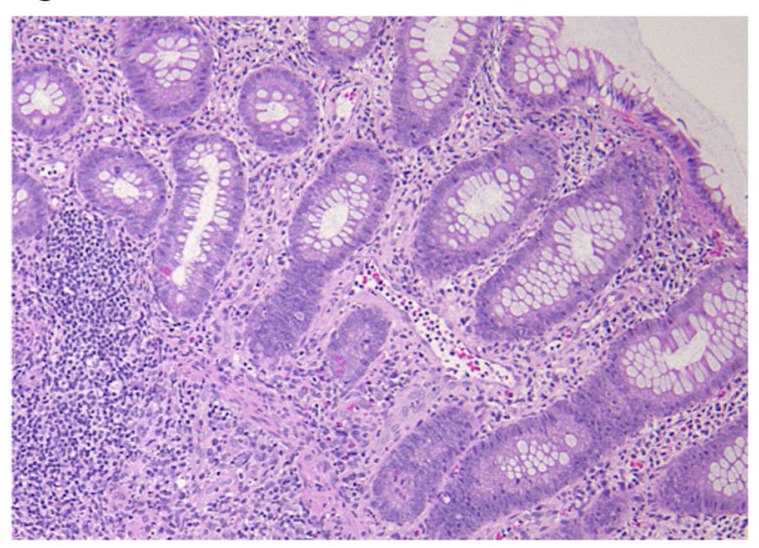

B

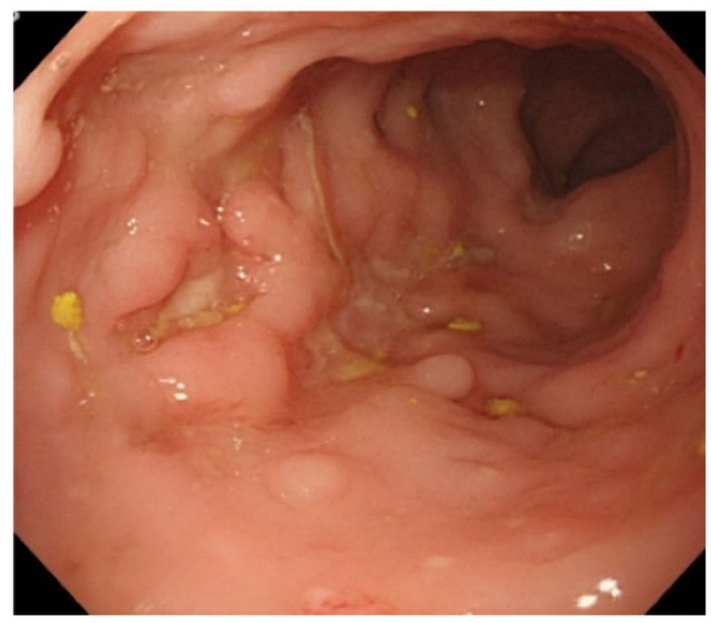

D

TNFAIP3:c.305A >G

(p. Asn102Ser)

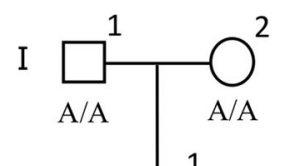

II

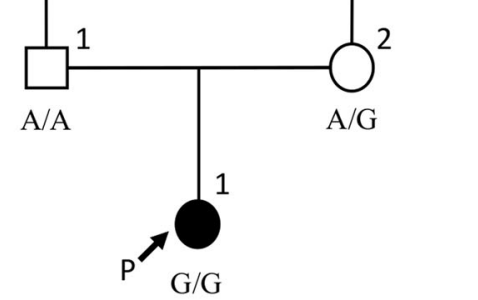

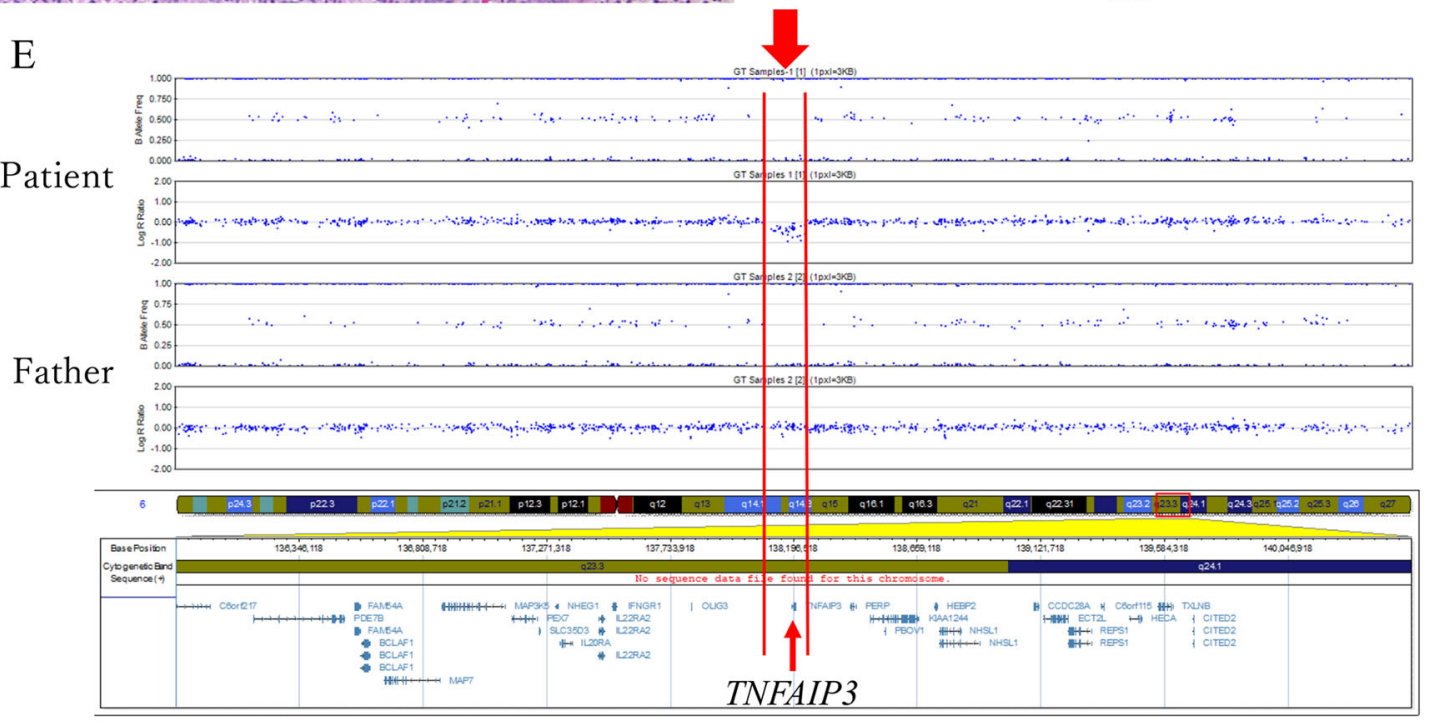

Fig. 1 Clinical data of the patient and the TNFAIP3 genotype in her family. A Severe perianal fistulae. B Punched-out ulcers in the rectum. C Mild diffuse neutrophilic inflammation, Paneth cell metaplasia and apoptosis in the descending colon (hematoxylin and eosin stain; $n \times 200$ ). D Pedigree chart of the TNFAIP3 genotype (NM_001270507.2(TNFAIP3):C.305A>G) in the proband, her parents, and her grandparents. E Singlenucleotide polymorphism array of the patient and her father. Both results showed that only the patient harbored the $119 \mathrm{~kb}$ deletion, including only TNFAIP3. 


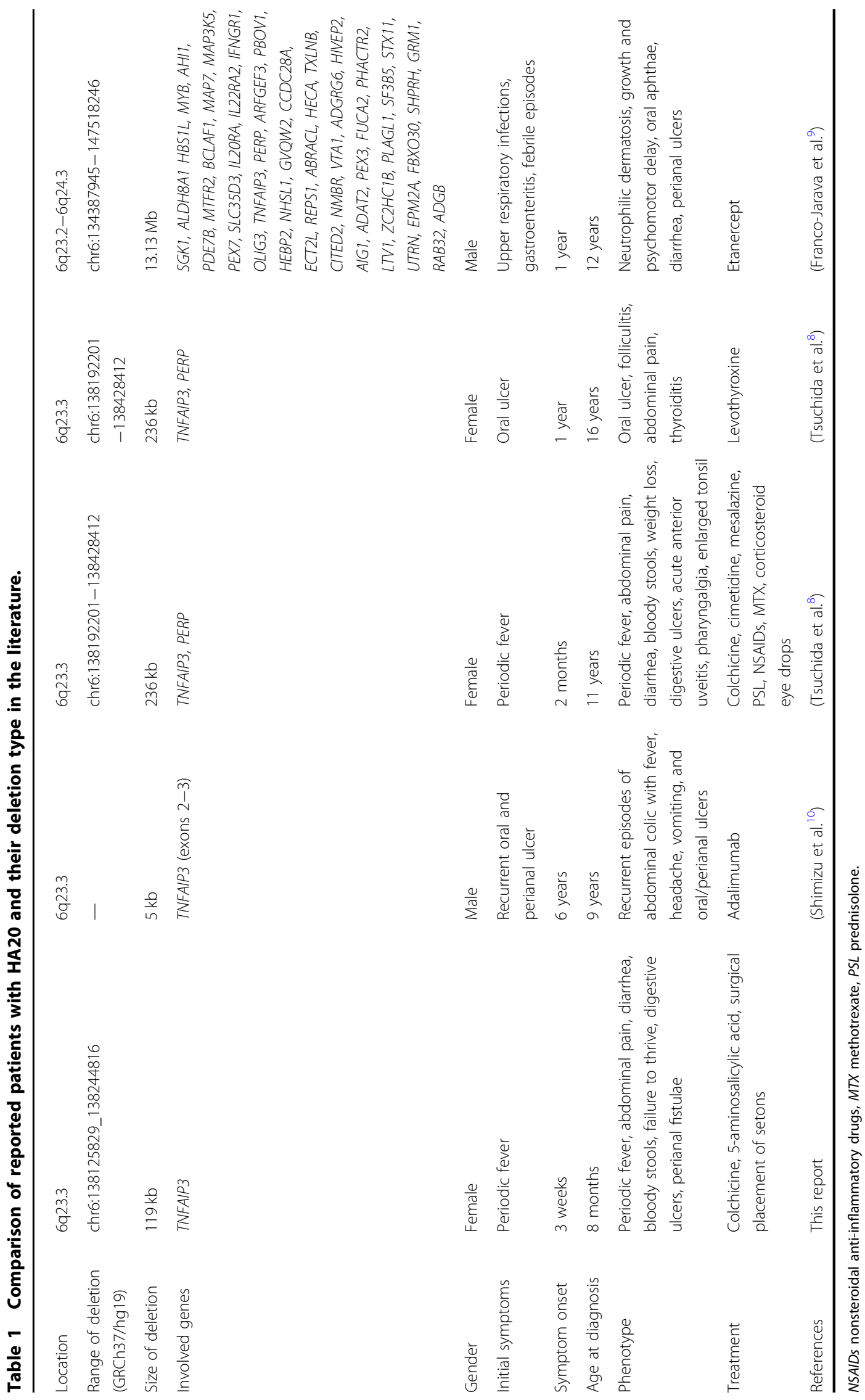


above the dentate line. Setons were placed to drain each fistula. Although the recurrent oral aphthous ulcers, arthritis, and intestinal punched-out ulcers observed in this patient met the International or Japanese criteria for diagnosing Behçet disease, these symptoms did not fulfill the criteria for the complete or incomplete type of Behçet disease. Colonic histopathology showed mild diffuse inflammation that was primarily neutrophilic, Paneth cell metaplasia, and crypt distortion throughout the colon; further, apoptosis was observed in the sigmoid colon and descending colon. A granuloma was also noted in the hepatic flexure. Despite macroscopic inflammation, no crypt atrophy or basal plasmacytosis was present (Fig. 1C).

The study was approved by the Institutional Review Board (IRB) of the National Center for Child Health and Development (IRB number: 926). Infantile-onset IBD with perianal lesions is a well-known manifestation of monogenic IBD, particularly in patients with IL10 signaling defects $^{4}$; therefore, we performed WES to identify the genetic etiology of the disease as described previously ${ }^{5}$ after obtaining written informed consent from the patient's family. DNA was extracted from the peripheral blood leukocytes of the patient, her parents, and her grandparents. Among the candidate variant genes, TNFAIP3 was notable, as it had been previously reported to be associated with monogenic IBD. We could not detect any homozygous IL1O and ILIOR variants suspected as pathogenic in the present patient. The patient was homozygous (A/A) for TNFAIP3 (c.305A > G; NM_001270507.2). This variant was also observed in the heterozygous state $(\mathrm{A} / \mathrm{G})$ in her mother (II-2) and is relatively common $(\mathrm{MAF}=0.0117)$ in the Japanese population ${ }^{6}$. However, her father (II-1) was homozygous for the reference allele (G/G) (Fig. 1D), suggesting that the patient had a deletion in this region that includes TNFAIP3. Subsequent CNV analysis conducted with WES data using EXCAVATOR ${ }^{7}$ revealed that the patient had only a single de novo $187 \mathrm{~kb}$ microdeletion within the TNFAIP3 gene (Supplementary Fig. S1). Genome-wide single-nucleotide polymorphism array analysis of the patient and her father using the Infinium Asian Screening Array-24 v1.0 BeadChip Kit (Illumina, San Diego, CA, USA) validated that the patient had a de novo $119 \mathrm{~kb}$ microdeletion involving only TNFAIP3 (arr[GRCh37] 6q23.3(138125829_138244816) $\times 1$, accession number SCV001424911 in ClinVar) (Fig. 1E). Both WES and CNV analyses indicated that the patient had lost the paternal allele of the TNFAIP3 gene, leading to HA20.

We detected a novel de novo microdeletion in the TNFAIP3 gene region of a patient with infantile-onset IBD complicated by a severe perianal lesion. The present case suggests that HA20 caused by TNFAIP3 heterozygous microdeletion should be considered when determining the cause of infantile-onset IBD with perianal lesions.
The majority of prior studies on VEO-IBD associated with HA20 have reported point mutations ${ }^{3}$. However, including this case, recent studies have revealed several TNFAIP3 deletions in patients with HA20, and these are summarized in Table $1^{8-10}$. Based on a comprehensive case review ${ }^{3}$, nine other reports ${ }^{8-16}$, and the present study, five of $69(7.2 \%)$ patients diagnosed with HA20 carried a deletion predicted to result in HA20. As approximately $7 \%$ of HA20 cases appear to be caused by deletions in TNFAIP3, we propose that $\mathrm{CNV}$ analysis should be included in the genetic testing for monogenic IBD, especially when suspecting HA20. However, Shimizu et al. reported a case possessing a very small deletion of only $5 \mathrm{~kb}$ in TNFAIP3; therefore, it is necessary to develop a smaller CNV detection algorithm using WES data to detect TNFAIP3 microdeletion.

For her infantile-onset perianal lesion, seton drainages were required for 1 year after their placement. Although infantile-onset IBD with perianal lesions is a well-known manifestation of monogenic IBD, particularly in patients with IL10 signaling defects ${ }^{4}$, this patient did not have any homozygous IL10 and IL1OR mutations despite low serum IL10 levels. Some HA20 cases show perianal lesions, but their severity is variable, and cases with severe perianal fistula, as in this case, are very rare. As VEO-IBD cases undergoing WES are increasing ${ }^{8,12-15}$, more cases including the deletion type will be accumulated in the future. It is also expected that the phenotype-genotype correlation will be elucidated. Overall, HA20 should be included as a differential diagnosis for infantile-onset IBD with perianal lesions, and CNV analysis in addition to WES should be considered to evaluate the TNFAIP3 deletion.

In conclusion, patients with HA20 caused by TNFAIP3 microdeletions can present infantile-onset IBD with severe perianal lesions. New genomic technologies to analyze microdeletions should be used to further investigate previously undiagnosed cases of monogenic IBD.

\section{HGV database \\ The relevant data from this Data Report are hosted at the Human Genome Variation Database at https://doi.org/10.6084/m9.figshare.hgv.2903.}

\section{Acknowledgements}

We would like to extend our sincere appreciation to the late Dr. Masanori Tanaka for his histopathological evaluation of this case. This research was (partially) supported by grants 19ek0109301s0202 and 19kk0205012s0404 from the Japan Agency for Medical Research and Development (AMED) and grants 2019A-3 and A-4 from the National Research Institute for Child Health and Development (NCCHD).

\section{Author details}

${ }^{1}$ Department of Maternal-Fetal Biology, National Research Institute for Child Health and Development, Tokyo 157-8535, Japan. ${ }^{2}$ Department of Gastrointestinal and Pediatric Surgery, Mie University Graduate School of Medicine, Tsu, Mie 514-8507, Japan. ${ }^{3}$ Division of Gastroenterology, National Center for Child Health and Development, Tokyo 157-8535, Japan.

${ }^{4}$ Department of Pediatrics, St. Marianna University School of Medicine,

Kanagawa 216-8511, Japan. ${ }^{5}$ Department of Anatomic Pathology, Hirosaki University Graduate School of Medicine, Aomori 036-8562, Japan. 'Department 
of Pediatrics, Mie University Graduate School of Medicine, Tsu, Mie 514-8507, Japan

\section{Conflict of interest}

The authors declare that they have no conflict of interest.

\section{Publisher's note}

Springer Nature remains neutral with regard to jurisdictional claims in published maps and institutional affiliations.

Supplementary information is available for this paper at https://doi.org/ 10.1038/s41439-020-00128-4.

Received: 25 June 2020 Revised: 1 September 2020 Accepted: 1 September 2020.

Published online: 14 January 2021

\section{References}

1. Shim, J. O. Recent advance in very early-onset inflammatory bowel disease. Intest. Res. 17, 9-16 (2019).

2. Zhou, Q. et al. Loss-of-function mutations in TNFAIP3 leading to A20 haploinsufficiency cause an early-onset autoinflammatory disease. Nat. Genet. 48, 67-73 (2016)

3. Berteau, F. et al. Autosomic dominant familial Behcet disease and haploinsufficiency A20: a review of the literature. Autoimmun. Rev. 17, 809-815 (2018).

4. Kotlarz, D. et al. Loss of interleukin-10 signaling and infantile inflammatory bowel disease: implications for diagnosis and therapy. Gastroenterology 143, 347-355 (2012).
5. Sato, T. et al. Novel TFAP2A mutation in a Japanese family with Branchiooculo-facial syndrome. Hum. Genome Var. 5, 5 (2018).

6. Nagasaki, M. et al. Rare variant discovery by deep whole-genome sequencing of 1,070 Japanese individuals. Nat. Commun. 6, 8018 (2015).

7. D'Aurizio, R. et al. Enhanced copy number variants detection from wholeexome sequencing data using EXCAVATOR2. Nucleic Acids Res. 44, e154 (2016).

8. Tsuchida, N. et al. Haploinsufficiency of A20 caused by a novel nonsense variant or entire deletion of TNFAIP3 is clinically distinct from Behcet's disease. Arthritis Res. Ther. 21, 137 (2019).

9. Franco-Jarava, C. et al. TNFAIP3 haploinsufficiency is the cause of autoinflammatory manifestations in a patient with a deletion of $13 \mathrm{Mb}$ on chromosome 6. Clin. Immunol. 191, 44-51 (2018).

10. Shimizu, M. et al. Haploinsufficiency of A20 with a novel mutation of deletion of exons 2-3 of TNFAIP3. Mod. Rheumatol. 1-5. https://doi.org/10.1080/ 14397595.2020.1719595 (2020). [E-pub ahead of print].

11. Zheng, $C$. et al. Infantile onset intractable inflammatory bowel disease due to novel heterozygous mutations in TNFAIP3 (A20). Inflamm. Bowel Dis. 24, 2613-2620 (2018)

12. Kim, H. Y. et al. The first case of an infant with familial $A 20$ haploinsufficiency in Korea. J. Korean Med. Sci. 35, e252 (2020).

13. Hautala, T. et al. A family with A20 haploinsufficiency presenting with novel clinical manifestations and challenges for treatment. J. Clin. Rheumatol. https:// doi.org/10.1097/RHU.0000000000001268 (2020). [Ahead of print].

14. Chen, Y. et al. A20 haploinsufficiency in a Chinese patient with intestinal Behcet's disease-like symptoms: a case report. Front. Immunol. 11, 1414 (2020).

15. Li, G. M. et al. Expanding the spectrum of A20 haploinsufficiency in two Chinese families: cases report. BMC Med. Genet. 20, 124 (2019).

16. Horita, N., Gul, A., Aksentijevich, I., Kastner, D. \& Remmers, E. F. Pseudodominance of autoinflammatory disease in a single Turkish family explained by coinheritance of haploinsufficiency of A20 and familial Mediterranean fever. Clin. Exp. Rheumatol. 37(Suppl 121), 89-92 (2019). 